\title{
Mechanical and electronic properties of SiC nanowires: an $a b$ initio study
}

J. B. Oliveira, ${ }^{1,2}$ J. M. Morbec, ${ }^{3, a)}$ and R. H. Miwa ${ }^{1}$

1) Instituto de Física, Universidade Federal de Uberlândia, Caixa Postal 593, CEP 38400-902, Uberlândia, MG, Brazil

2) Instituto Federal do Triângulo Mineiro, CEP 38305-200, Ituiutaba, MG, Brazil

${ }^{3)}$ Faculty of Physics, University of Duisburg-Essen, Lotharstrasse 1, 47057 Duisburg, Germany

(Dated: 24 October 2019)

Using first-principles calculations, based on density functional theory, we have investigated the mechanical and electronic properties of hydrogen-passivated 3C-, $2 \mathrm{H}-, 4 \mathrm{H}-$, and $6 \mathrm{H}-\mathrm{SiC}$ nanowires, analyzing the effects of the diameter on these properties. Our results show that the band-gap energies of the nanowires are larger than the corresponding bulk values, and decrease with the increasing diameter. All nanowires investigated exhibit direct band gaps, in contrast with the indirect band gaps observed in bulk SiC. The effect of uniaxial stress on the electronic properties of $\mathrm{SiC}$ nanowires has been also examined, and our results reveal that the band-gap dependence on the strain is different for each nanowire polytype. In 3C-SiC nanowires, the band gaps increase (decrease) with tensile (compressive) strain. For $4 \mathrm{H}-$ and $6 \mathrm{H}-\mathrm{SiC}$ nanowires the influence of strain on the band gaps is more pronounced in the thicker wires. Finally, we estimated the band offsets of hypotetical NW homostructures, composed by stacking SiCNW layers with different polytypes.

PACS numbers: $62.25 .-\mathrm{g}, 73.22 . \mathrm{f}, 71.15 . \mathrm{Mb}, 71.15 . \mathrm{Nc}$

\section{INTRODUCTION}

One-dimensional silicon carbide $(\mathrm{SiC})$ nanostructures have attracted increasing attention in the last years owing to their great potential for nanotechnology applications. These nanostructures combine the outstanding properties of $\mathrm{SiC}$ (high thermal conductivity, excellent chemical stability, high electron drift velocity, high breakdown electric field, good field-emitting performance, and others) with quantum-size effects, and are promising materials for the fabrication of electronic and optoelectronic nanodevices.

Among the different $\mathrm{SiC}$ nanostructures, $\mathrm{SiC}$ nanowires (NWs) are particularly interesting because they exhibit exceptional optical, electronic, chemical, and mechanical properties. These NWs have been synthesized using different methods in recent years ${ }^{1-8}$ and are expected to have great potential for use in lightemitting diodes and field-emission displays due to their good photoluminescence ${ }^{9-11}$ and field-emission ${ }^{3,7,12,13}$ properties. Moreover, SiCNWs present high strength and elasticity, ${ }^{14}$ besides an unusual large strain plasticity at low temperatures, ${ }^{15}$ and could be used as reinforcing elements in composite materials. ${ }^{16,17}$

Many characteristics of the SiCNWs are strongly related to their low dimensionality. For this reason, it is important to study the effects of quantum confinement on the properties of these systems. Rurali ${ }^{18}$ investigated (using first-principles calculations) the electronic properties of pure and hydrogen-passivated 3CSiCNWs ${ }^{19}$ grown along the [110] direction, and showed that pure NWs present metallic character due to surface

\footnotetext{
a) Electronic mail: jmmorbec@gmail.com
}

reconstructions, whereas quantum confinement induces a gap-broadening effect in hidrogenated NWs. In another theoretical work, Durandurdu ${ }^{20}$ observed that the band gaps of pure wurtzite SiCNWs increase with increasing diameters, in contrast to hydrogenated wurtzite SiCNWs whose band gaps decrease with increasing diameters. Using first-principles calculations, Yan et al. ${ }^{21}$ investigated the effect of uniaxial stress on the electronic properties of hydrogen-passivated [111] 3C-SiCNWs. They verified that these NWs have direct band gaps, and that their band gaps increase with increasing tensile stress or with decreasing diameters. Meanwhile, Wang et al. ${ }^{22}$ performed first-principles calculations to study the energetic stability and electronic properties of hydrogenated [110]-, [111]-, and [112]-oriented 3C-SiCNWs, and [0001]oriented $2 \mathrm{H}-\mathrm{SiCNW}$. They verified that the energetic stability of the SiCNWs depends on their orientation and that $3 \mathrm{C}-\mathrm{SiCNW}$ oriented along the [111] direction are the most favorable ones. In addition, they observed that the band gaps of the NWs decrease with increasing diameters, and that [112]-oriented 3C-SiCNWs have indirect band gaps (similar to bulk $\mathrm{SiC}$ ), while the other NWs have direct band gaps.

In this work we have investigated, using ab initio calculations, the effects of the reduced dimensionality on the electronic and mechanical properties of SiCNWs. We considered SiCNWs constructed from the cubic (3C or $\beta$ ) and hexagonal $(2 \mathrm{H}, 4 \mathrm{H}, 6 \mathrm{H})$ polytypes, oriented along the [111] and [0001] directions, respectively. We have analyzed the dependence of the Young's moduli and the band-gap energies of the nanowires on their diameters, as well as the influence of uniaxial stress on their electronic properties. By considering a set of stacking sequences of different $\mathrm{SiC}$ polytypes, we estimate the band offsets of hypothetical SiCNW homostructures. 


\section{COMPUTATIONAL METHODS}

Our calculations were performed within the framework of the density functional theory (DFT), ${ }^{23}$ as implemented in the SIESTA code, ${ }^{24}$ employing norm-conserving fully separable Troullier-Martins pseudopotentials ${ }^{25}$ to describe the electron-ion interactions and the local density approximation (LDA) $)^{26,27}$ for the exchange-correlation functional. The Kohn-Sham orbitals were expanded in a linear combination of numerical pseudoatomic orbitals, ${ }^{28}$ with an energy shift of $0.20 \mathrm{eV}$. We used a double-zeta basis set for $\mathrm{H}$ atoms ( $\mathrm{H}$ atoms were employed to passivate the surface dangling bonds) and double-zeta with polarization functions for $\mathrm{Si}$ and $\mathrm{C}$ atoms. A mesh cutoff energy of $170 \mathrm{Ry}$ was used for the real space grid integration. The nanowires were modeled within the supercell approach, using a vacuum region of about $10 \AA$ between the wires in the radial direction. All the atomic positions were allowed to relax during the geometry optimization until the Hellman-Feynman forces were less than $0.05 \mathrm{eV} / \AA$ for $2 \mathrm{H}-\mathrm{SiCNW}$ and $0.02 \mathrm{eV} / \AA$ for the other NWs. The Brillouin zone was sampled using $1 \mathbf{k}$ points for $3 \mathrm{C}-, 4 \mathrm{H}-$, and $6 \mathrm{H}-\mathrm{SiCNW}$, and $2 \mathrm{k}$-points for $2 \mathrm{H}-\mathrm{SiCNW}$. The convergence of the $k$-point grid was examined for the 3C-SiCNWs: considering up to $14 k$ points, we did not find any change in the cohesive energy or in the band gap of these systems.

\section{RESULTS AND DISCUSSION}

\section{A. SiC bulk}

We initially examined the structural, mechanical, and electronic properties of bulk 3C-, 2H-, 4H-, and 6H-SiC. Our results for optimized lattice constants, Young's moduli, band gap energies, and cohesive energies are presented in Table I. The cohesive energies per atom, $E_{c}$, and the Young's moduli, $Y$, were estimated using the equations,

$$
E_{c}=\frac{\sum_{i} n_{i} E_{i}-E}{\sum_{i} n_{i}}
$$

and

$$
Y=\frac{1}{V}\left(\frac{d^{2} E}{d \epsilon^{2}}\right)_{\epsilon=0},
$$

where $E$ is the total energy, $n_{i}$ is the number of atoms of specie $i(i=\mathrm{Si} / \mathrm{C}$ for bulk, and $i=\mathrm{Si} / \mathrm{C} / \mathrm{H}$ for $\mathrm{NW}), E_{i}$ is the energy of the isolated atom, $\epsilon$ is the strain, and $V$ is the equilibrium volume.

Our calculated cohesive energies (Table I) indicate that the $2 \mathrm{H}$ structure (which has the lowest $E_{c}$ ) is the most unstable structure among the polytypes investigated, whereas $4 \mathrm{H}$ and $6 \mathrm{H}$ are the most stable ones. This is consistent with previous theoretical works ${ }^{36,37}$ and with the fact that the $2 \mathrm{H}$ polytype is rarely observed. We noted that our result for $E_{c}$ of bulk $3 \mathrm{C}-\mathrm{SiC}$ $\left(E_{c}^{3 \mathrm{C}}=6.778 \mathrm{eV} /\right.$ atom $)$ is larger than the experimental value, $6.34 \mathrm{eV} /$ atom $^{32}$ [see Table I]; comparing with previous theoretical studies, (i) Chang and Cohen ${ }^{38}$ obtained $E_{c}^{3 \mathrm{C}}=6.66 \mathrm{eV} /$ atom, and (ii) Jiang et al. ${ }^{39}$ obtained $6.40 \mathrm{eV} /$ atom, both calculations were performed within the DFT approach, the former using plane waves as a basis set, while the latter used atomic orbitals.

In our electronic-structure calculations, we found indirect band-gaps of $1.31,2.69,2.20$, and $2.00 \mathrm{eV}$ for bulk $3 \mathrm{C}-, 2 \mathrm{H}-, 4 \mathrm{H}-$, and $6 \mathrm{H}-\mathrm{SiC}$, respectively. These results are underestimated by more than $30 \%$ with respect to the experimental values [see Table I], which is common in DFT calculations, but are in good agreement with previous theoretical results: for example, using DFT calculations (i) Persson and Lindefelt ${ }^{41}$ found 1.30, 2.11, 2.17 and 1.97 for $E_{g}$ of bulk 3C-, 2H-, 4H-, and $6 \mathrm{H}-\mathrm{SiC}$, respectively, and (ii) Wang et al. ${ }^{42}$ obtained 1.37, 2.78, 2.36 , and $2.14 \mathrm{eV}$.

\section{B. SiC nanowires}

\section{Cohesive energy and Young's modulus}

Next, we studied 3C-SiCNWs oriented along the [111] direction, and $2 \mathrm{H}-, 4 \mathrm{H}-$, and $6 \mathrm{H}-\mathrm{SiCNW}$ s oriented along the [0001] direction. In order to investigate the diameter dependence of the electronic and mechanical properties of these systems, we considered NWs with different diameters, $d$, as depicted in Fig. 1. We studied 3C-, 2H-, $4 \mathrm{H}$ - and $6 \mathrm{H}-\mathrm{SiCNWs}$ with $d \approx 0.71,1.07$, and $1.42 \mathrm{~nm}$. The dangling bonds at the nanowire surfaces were passivated by $\mathrm{H}$ atoms to eliminate the surface states and prevent surface reconstruction. We found optimized Si$\mathrm{C}$ bond lengths from 1.87 to $1.90 \AA$; the $\mathrm{Si}-\mathrm{H}$ and $\mathrm{C}-\mathrm{H}$ bond lengths at the surfaces are about 1.52 and $1.12 \AA$, respectively.

The cohesive energy of each nanowire $\left(E_{c}^{\mathrm{NW}}\right)$, calculated using Eq. (1), is presented in Table II. For all types of nanowires, the values of $E_{c}^{\mathrm{NW}}$ are smaller than the corresponding bulk values $\left(E_{c}^{\text {bulk }}\right)$ [see also Table I], but increase and approach to $E_{c}^{\text {bulk }}$ with increasing diameters. We also noted that the $2 \mathrm{H}-\mathrm{SiCNW}$ s have the largest cohesive energies, which suggests that $2 \mathrm{H}-\mathrm{SiCNW}$ are more stable than 3C-, 4H-, and 6H-SiCNWs with similar diameters. This result contrasts with that obtained for bulk $\mathrm{SiC}$ [Table I] that indicates that $2 \mathrm{H}$ is the less stable polytype. Our results are consistent with the theoretical work of Ito et al. ${ }^{43}$ in which $2 \mathrm{H}$ was predicted to be the most stable structure for SiCNWs with $d<20 \mathrm{~nm}$, but do not agree with the $a b$ initio study performed by Wang et al. ${ }^{22}$ in which $\mathrm{SiC}$ nanowires with $d<2.8 \mathrm{~nm}$ were investigated and [111] 3C-SiCNWs were found to be more stable than [0001] 2H-SiCNWs. Ito et al. ${ }^{43}$ attributed the instability of the 3C-SiCNWs with $d<20 \mathrm{~nm}$ to the large number of dangling bonds at their surfaces, when 
TABLE I. Calculated equilibrium lattice constants, bulk moduli $\left(B_{o}\right)$, band gap energies $\left(E_{g}\right)$, and cohesive energies $\left(E_{c}^{\text {bulk }}\right)$ of bulk 3C-, $2 \mathrm{H}-, 4 \mathrm{H}-$, and $6 \mathrm{H}-\mathrm{SiC}$. The values in parentheses are experimental data taken from Refs. $31-33$.

\begin{tabular}{lcccc}
\hline \hline & lattice constants $(\AA)$ & $B_{o}(\mathrm{GPa})$ & $E_{g}(\mathrm{eV})$ & $E_{c}^{\mathrm{bulk}}(\mathrm{eV} / \mathrm{atom})$ \\
\hline $3 \mathrm{C}-\mathrm{SiC}$ & $a=4.35\left(4.36^{\mathrm{a}}\right)$ & $219.1(224.0)$ & $2.31\left(2.39^{\mathrm{a}}\right)$ & $6.778\left(6.34^{\mathrm{b}}\right)$ \\
$2 \mathrm{H}-\mathrm{SiC}$ & $a=3.08\left(3.08^{\mathrm{a}}\right)$ & $207.8(223.4)$ & & \\
& $c=5.02\left(5.05^{\mathrm{a}}\right)$ & $2.69\left(3.33^{\mathrm{a}}\right)$ & \\
$4 \mathrm{H}-\mathrm{SiC}$ & $a=3.07\left(3.07^{\mathrm{a}}\right)$ & & & \\
& $c=10.07\left(10.05^{\mathrm{a}}\right)$ & $209.0(223.4)$ & $2.200\left(36^{\mathrm{a}}\right)$ & 7.208 \\
$6 \mathrm{H}-\mathrm{SiC}$ & $a=3.08\left(3.08^{\mathrm{a}}\right)$ & & \\
& $c=15.09\left(15.12^{\mathrm{a}}\right)$ & & & 7.208 \\
\hline \hline
\end{tabular}

\footnotetext{
${ }^{\text {a }}$ Ref. 31
}

b Refs. 32,33
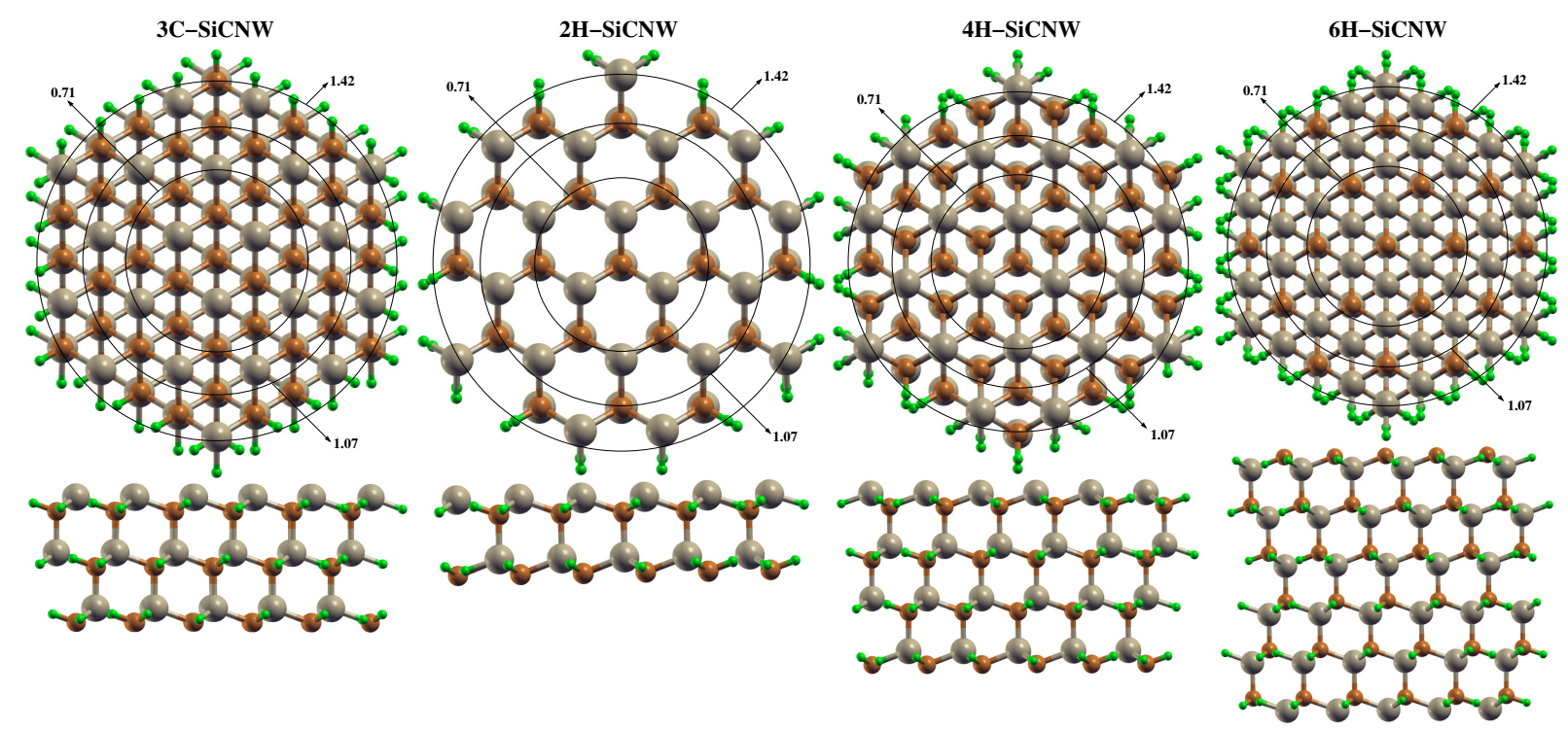

FIG. 1. (Color online) Cross-section (top panel) and side (bottom panel) views of the 3C-SiCNWs oriented along the [111] direction, and $2 \mathrm{H}-, 4 \mathrm{H}-$, and $6 \mathrm{H}-\mathrm{SiCNW}$ s oriented along the [0001] direction. The circles indicate the different diameters considered in each case (the diameters are in $\mathrm{nm}$ ). Side view is shown for the thickest nanowire.

compared to $2 \mathrm{H}-\mathrm{SiCNW}$. Here we found, however, that $3 \mathrm{C}$-SiCNWs are also less stable than $2 \mathrm{H}$-SiCNWs when the surface dangling bonds are passivated by $\mathrm{H}$ atoms. The reduction of the cohesive energy in the NWs can be attributed to the formation of surface dangling bonds, which is proportional to the NW surface area $(\propto d)$. Whereas the $\mathrm{SiC}$ bulk contribution, which is proportional to the NW volume $\left(\propto d^{2}\right)$, increases the cohesive energy, being $E_{c}^{\text {bulk }}$ its upper limit, that is, we can infer that the former contribution reduces for larger diamenters, where $E_{c}^{\mathrm{NW}} \rightarrow E_{c}^{\mathrm{bulk}}$.

In Table II, we also present our results for the Young's moduli of SiCNWs, determined according to Eq. (2), with strains from $-4 \%$ to $+4 \%$ along the axial direction. We note that, for all nanowires investigated, the Young's moduli decrease with the increasing diameter. This behavior is similar to that observed in $\mathrm{Ag}^{44,45}, \mathrm{~Pb}^{45}$, and (more recently) for $\mathrm{GaAs}^{46}$ nanowires, but is op- posite to that found in $\operatorname{InAs},{ }^{47} \mathrm{InP},{ }^{47} \mathrm{Si}^{48}$ ? and $\mathrm{Ge}^{50}$ nanowires. There is a dependence of the Young's moduli on the nanowire diameter, which is larger for the hexagonal nanowires (the Young's modulus of $2 \mathrm{H}-, 4 \mathrm{H}-$ and $6 \mathrm{H}-$ SiCNWs reduces by $\sim 12,15$, and $19 \%$ by increasing the NW diameter from 0.71 to $1.42 \mathrm{~nm}$ ). Similar dependence between the Young's modulus and the NW diameter has been observed for GaAs NWs: Chen et al. ${ }^{46}$ found a reduction of $\sim 15 \%(189 \rightarrow 161 \mathrm{GPa})$ in the Young's modulus of wurtzite GaAs NWs by increasing the NW diameter from 75 to $170 \mathrm{~nm}(\sim 126 \%)$. Meanwhile, as shown in Table II, the Young's modulus of 3C-SiCNW is less sensitive to the diameter: while the diameter increases by a factor of $2(0.71 \rightarrow 1.42 \mathrm{~nm})$, the Young's modulus decreases only by about $5.3 \%(642 \rightarrow 608 \mathrm{GPa})$. This behavior observed for $3 \mathrm{C}-\mathrm{SiCNW}$ is consistent with previous theoretical works, ${ }^{21,51}$ and our calculated Young's moduli for $3 \mathrm{C}-\mathrm{SiCNW}$ are in good agreement with the values 
TABLE II. Calculated cohesive energies $\left(E_{c}^{\mathrm{NW}}\right)$, band gap energies $\left(E_{g}\right)$, and Young's moduli $(Y)$ for $3 \mathrm{C}-, 2 \mathrm{H}-, 4 \mathrm{H}-$, and $6 \mathrm{H}-\mathrm{SiCNW}$ as functions of diameter $d$.

\begin{tabular}{lccc}
\hline \hline$d(\mathrm{~nm})$ & $E_{c}^{\mathrm{NW}}(\mathrm{eV} /$ atom $)$ & $E_{g}(\mathrm{eV})$ & $Y(\mathrm{GPa})$ \\
\hline 3C-SiCNW & & & \\
\hline 0.71 & 5.07 & 3.24 & 642.03 \\
1.07 & 5.49 & 2.62 & 617.70 \\
1.42 & 5.72 & 2.17 & 608.00
\end{tabular}

\begin{tabular}{llll}
$2 \mathrm{H}-\mathrm{SiCNW}$ & & & \\
\hline 0.71 & 5.30 & 3.94 & 581.21 \\
1.07 & 5.61 & 3.29 & 523.24 \\
1.42 & 6.17 & 2.97 & 494.60
\end{tabular}

\begin{tabular}{llll}
$4 \mathrm{H}-\mathrm{SiCNW}$ & & & \\
\cline { 1 - 1 } 0.71 & 5.22 & 3.90 & 563.65 \\
1.07 & 5.35 & 3.39 & 494.14 \\
1.42 & 5.70 & 3.02 & 480.59 \\
& & & \\
$6 \mathrm{H}-\mathrm{SiCNW}$ & & & \\
0.71 & 5.07 & 3.77 & 584.56 \\
1.07 & 5.35 & 3.11 & 540.08 \\
1.42 & 5.71 & 2.76 & 472.33 \\
\hline \hline
\end{tabular}

obtained by Yan et $a .^{21}$ using DFT calculations. Our calculated Young's moduli for the nanowires are larger than $300 \mathrm{GPa}$, which indicates that $\mathrm{SiC}$ nanowires are more rigid than $\mathrm{Si}^{48,49}$ and $\mathrm{Ge}^{50}$ nanowires grown along the same direction and with similar diameters.

In NW systems, we can infer that there are two contributions to the Young's moduli, one from the NW surface, and another from the inner (bulk) region of the NW. In this case, the relative contribution of each term is proportional to the surface/bulk ratio, namely $1 / d$. Within such a scenario, our results indicate that the NW surfaces contribute to increase the Young's moduli of SiCNWs, since the surface effects are strenghtened for NWs with smaller diameters. On the other hand, by increasing the NW diameter, the role played by the surface becomes less important, and the Young's moduli becomes ruled by the bulk region of the NW. However, it is worth noting that such a surface/bulk dependence is commited to the NW bulk material, and its surface properties. For instance, the Young's modulus of Ge (GaAs) NWs increases (decreases) by increasing the NW diameter. ${ }^{46,50}$

\section{Electronic properties}

Our electronic structure calculations reveal that all nanowires investigated have direct band gaps at $\Gamma$ point, in contrast with the indirect band gaps observed in bulk $\mathrm{SiC}$. We note that the band-gaps of the nanowires [Ta-
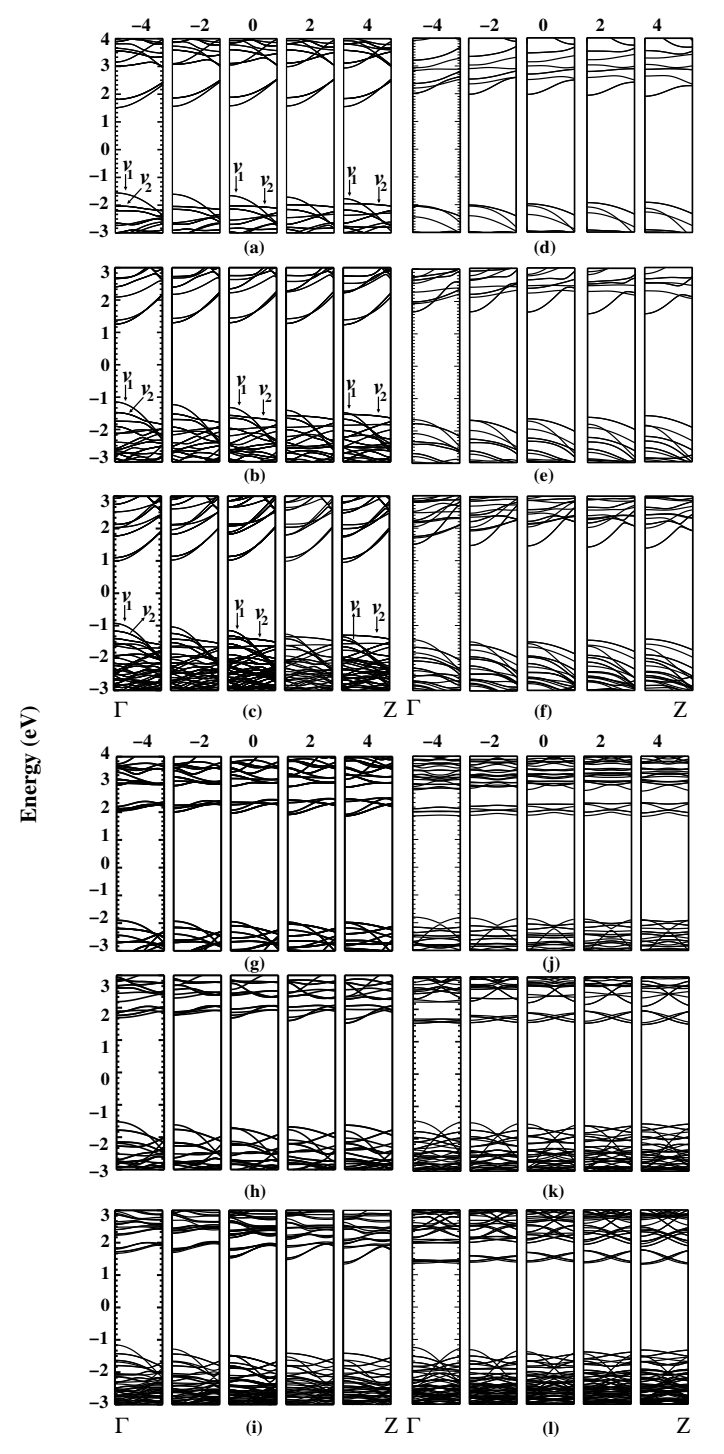

FIG. 2. Electronic band structures for (a)-(c) 3C-, (d)-(f) $2 \mathrm{H}-,(\mathrm{g})-(\mathrm{h}) 4 \mathrm{H}-$ and (j)-(l) $6 \mathrm{H}-\mathrm{SiCNW}$ sith diameters of $0.71,1.07$, and $1.42 \mathrm{~nm}$.

ble II] are larger than those of their counterpart bulk phases [see Table I]. These results indicate strong effects of the quantum confinement on the electronic properties of the nanowires. It is interesting to note that $3 \mathrm{C}$ is the structure with the narrowest band gap among the nanowires investigated, and also among the different polytypes for bulk SiC [see Table I]. Our results for 3Cand $2 \mathrm{H}-\mathrm{SiCNW}$ are consistent with previous theoretical works. $^{20-22}$

In addition, we have investigated the effects of strain on the electronic properties of SiCNWs. All nanowires were strained from $-4 \%$ (compressive) to $+4 \%$ (tensile) strain, with increments of $1 \%$. In Fig. 2 we present the electronic band structures of the SiCNWs for wave vector along 


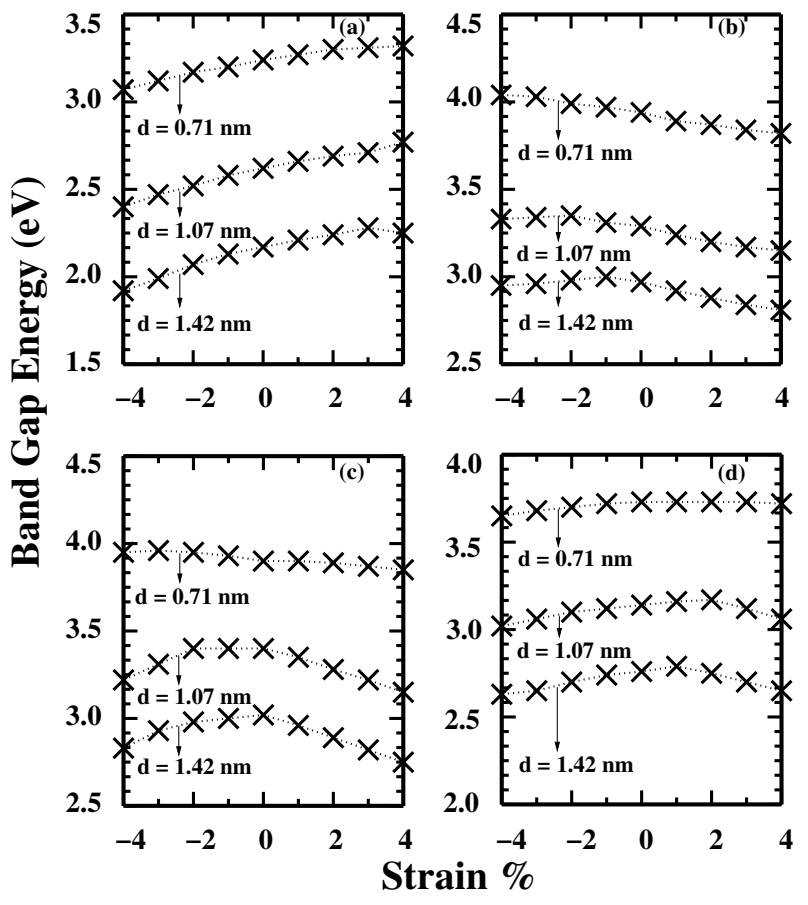

FIG. 3. Band gap energies (in eV) as a function of the strain (in \%) for (a) 3C-, (b) 2H-, (c) 4H-, and (d) 6H-SiCNWs.

the NW growth direction. We verify that, in general, the main features of the electronic band structure have been preserved upon external strain; however there are some changes that are worth being reported. The highest occupied states, at the $\Gamma$-point, of the the $3 \mathrm{C}$-SiCNW is characterized by a light-hole (LH) band followed by a heavy-hole (HH) band, $v 1$ and $v 2$ in Figs. $2(\mathrm{a})-2(\mathrm{c})$. The energy difference between $v 1$ and $v 2$ (at the $\Gamma$-point) increases (decreases) by compressing (stretching) the NW. For the thinnest 3C-SiCNW, even for a strain of $+4 \%, v 1$ lies above $v 2$; however, such an energy difference reduces by increasing the NW diameter. For the largest diameter, a valence band crossing between $v 1$ and $v 2$ takes place by stretching the NW. Indeed, similar behavior has been verified for the other SiCNWs. Those findings allow us to infer that, upon tensile strain, the hole mobility will be reduced, since the edge of the valence band becomes characterized by HH states $(v 2)$. On the other hand, by compressing the SiCNWs, we find LH states $(v 1)$ at edge of the valence band. For instance, by calculating the hole effective masses $\left(m_{h}^{*}\right)$, defined as $\hbar^{2}\left(\frac{\partial^{2} E}{\partial k^{2}}\right)^{-1}$, we obtained $m_{h}^{*} \approx 0.5 m_{0}$ ( $m_{0}$ is the free electron rest mass) for the 3C-SiCNW with a diameter of $1.42 \mathrm{~nm}$; by stretching the NW by $+4 \%, m_{h}^{*}$ increases to $5.3 m_{0}$. Focusing on the conduction bands, we verify that the lowest unoccupied states of $4 \mathrm{H}-$ and $6 \mathrm{H}-\mathrm{SiCNW}$ secome less localized by stretching the NWs from $-4 \%$ to $+4 \%$. For instance, in the $4 \mathrm{H}-\mathrm{SiCNW}$ (with $d=1.42 \mathrm{~nm}$ ) compressed by $-4 \%$, the lowest unoccupied states lie in an energy interval of $\sim 0.2 \mathrm{eV}$ [Fig. 2(h)]; while the strained NW (to $+4 \%$ ) presents an energy dispersion $\sim 0.6 \mathrm{eV}$, reducing the electron effective mass $\left(m_{e}^{*}\right)$. Here, $m_{e}^{*}$ reduces from $1.1 m_{0}$ (strain $\left.=-4 \%\right)$ to $0.3 m_{0}$ by applying a tensile strain (strain $>0$ ). Such a delocalization of the lowest unoccupied states has not been verified for the $3 \mathrm{C}$ - and 2H-SiCNWs, Figs. 2(a)-2(c), and 2(d)-2(f), respectively. The dispersion of those energy bands has been preserved for the different NW diameters and strains. Those systems present lower effective masses, weakly dependent on the external strain. For example, the $m_{e}^{*}$ of $3 \mathrm{C}-\mathrm{SiCNW}$ with $d=1.42 \mathrm{~nm}$ varies from $0.40 m_{0}$ to $0.36 m_{0}$ for external strain of $-4 \%$ to $+4 \%$, respectively.

As can be seen in Fig. 3, the dependence between the band-gap energies and the strain is different for each nanowire type. For example, we found that the band gaps of 3C-SiCNWs with $d \approx 0.71$ and $1.07 \mathrm{~nm}$ [Fig. 3(a)] exhibit a monotonic behavior, increasing (decreasing) with tensile (compressive) stress. On the other hand, the band gap energies of $2 \mathrm{H}-\mathrm{SiCNWs}$ [Fig. 3(b)] decrease with tensile stress $(0$ to $+4 \%)$. For the hexagonal $4 \mathrm{H}$ and $6 \mathrm{H}$ nanowires we note that the influence of strain on the band gaps is small in the thinnest nanowires and it is more pronounced in the thickest wires; for instance, the band gap of $4 \mathrm{H}-\mathrm{SiCNW}$ with $d \approx 0.71 \mathrm{~nm}$ decreases only by $1.28 \%$ under tensile strain of $4 \%$ [Fig. 3(c)], while the band gap of the thicker $4 \mathrm{H}-\mathrm{SiCNW}(d \approx 1.42 \mathrm{~nm})$ decreases by $8.94 \%$ under similar condition. In order to provide a more complete picture of those changes in the SiCNW band-gap as a function of the NW diameter and the external strain, we next compare the energy positions of the valence band maximum (VBM) and the conduction band minimum $(\mathrm{CBM})$, by considering a common energy reference. Here, following the procedure proposed by Beckman et al.,${ }^{57}$ the VBM and the CBM were lined up with respect to the energy position of the highest occupied state of an isolated $\mathrm{H}_{2}$ molecule within our supercell approach. Our results, presented in Fig. 4, show that (i) the increase of the energy gap in $3 \mathrm{C}-\mathrm{SiCNW}$, strained from $-4 \%$ to $+4 \%$, is mostly ruled by the downshift of the VBM, whereas the CBM is weakly perturbed [Fig. 4(a)]. In contrast, (ii) the downshift of the CBM by stretching the $2 \mathrm{H}-$ and $4 \mathrm{H}-\mathrm{SiCNW}$ from 0 to $+4 \%$ [Figs. 4 (b) and 4(c)] rules the band-gap reduction in those NWs; and (iii) for the $6 \mathrm{H}-\mathrm{SiCNW}$ with larger diameters (1.07 and $1.42 \mathrm{~nm}$ ), the increase of the energy gap by stretching the NW from $-4 \%$ to around $1 \%$ is mainly dictated by the changes on the energy position of the VBM.

Stacking faults (SF) play an important role in the electronic properties of $\mathrm{SiCNWs}^{39,58}$. In a recent experimental work, Kuang and $\mathrm{Cao}^{59}$ verified that SFs in 3CSiCNWs promote the electron-hole separation along the NW, since the formation the SFs are characterized by $2 \mathrm{H}$ polytypes embedded in the $3 \mathrm{C}$ host, namely SFs give rise to a homostructure of SiCNW composed by two different stacking sequences. They found a type-II band alignment for nanowires with diameters in the range of 50 to $200 \mathrm{~nm}$, with electrons/holes in the $3 \mathrm{C} / 2 \mathrm{H}$ regions. Type-II confinement has been also verified in homojunctions of InP NWs composed by a single layer of zincblend 


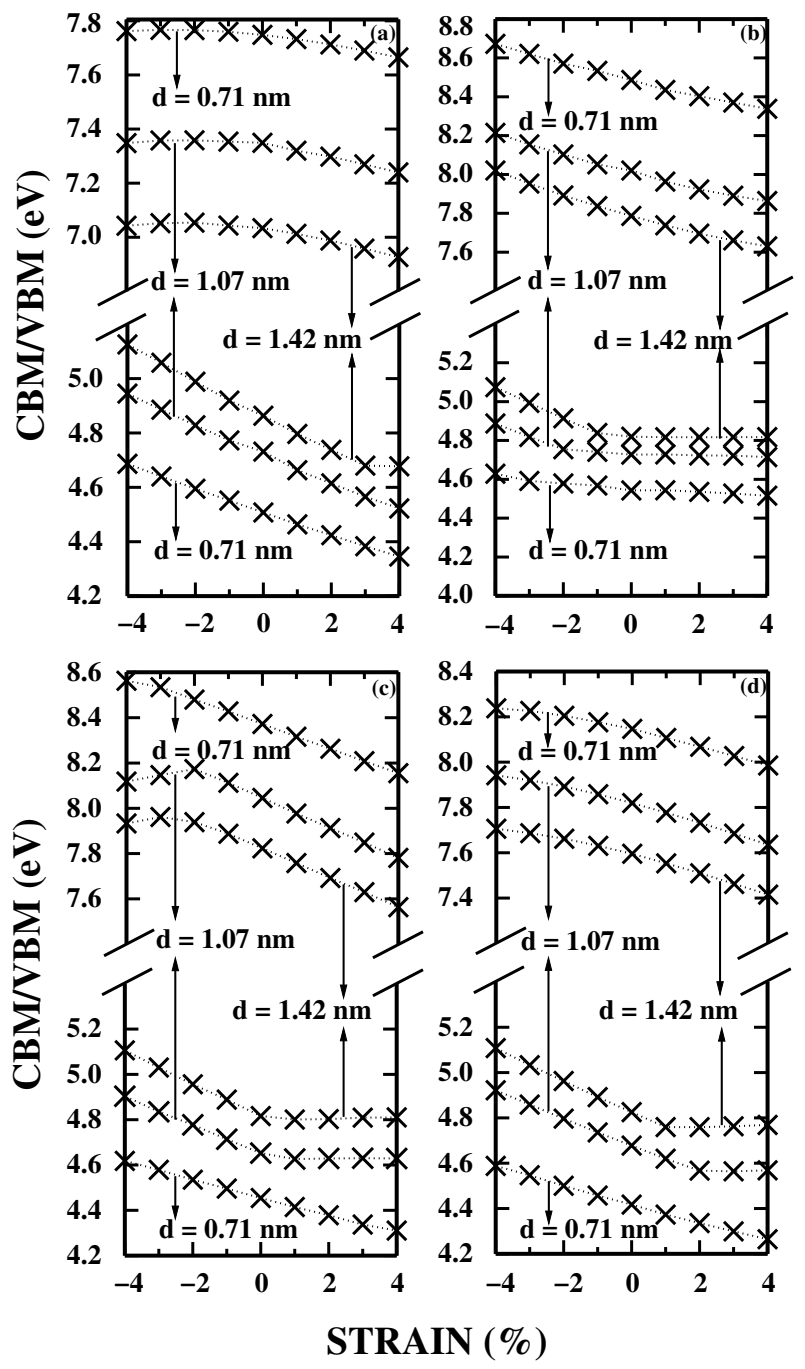

FIG. 4. Energy positions of the CBM and VBM as a function of the strain for (a) 3C-, (b) $2 \mathrm{H}-$, (c) $4 \mathrm{H}-$ and (d) $6 \mathrm{H}-\mathrm{SiCNW}$. The VBM and the CBM were lined up with respect to the energy position of the highest occupied state of an isolated $\mathrm{H}_{2}$ molecule within our supercell approach. ${ }^{57}$

(zb) region embedded in the $\mathrm{InP}$ wurtzite (wz) host. ${ }^{60}$ Further first-principles calculations supported the typeII band offset in wz/zb-InP NWs. ${ }^{61}$ The band alignment may depend, however, on the width of each stacking (polytype) region as well as on the NW diameter. Indeed, such a diameter dependence has been verified in a recent ab initio study ${ }^{62}$ on cubic/hexagonal Si NW homojunctions, in which type-I band alignment was found for nanowires with diameters of 1.0 and $1.8 \mathrm{~nm}$, whereas type-II band offset was obtained for thicker nanowires with diameter of $3.2 \mathrm{~nm}$.

Here, based on the energy positions of the VBM and CBM of the SiCNWs, as shown Fig. 4, we can infer the band offsets for different combinations of SiCNWs with similar diameters, namely giving rise to SiCNW homostructures composed by different stacking sequences. For NWs with $d \approx 1.07 \mathrm{~nm}$, for instance, the VBM of

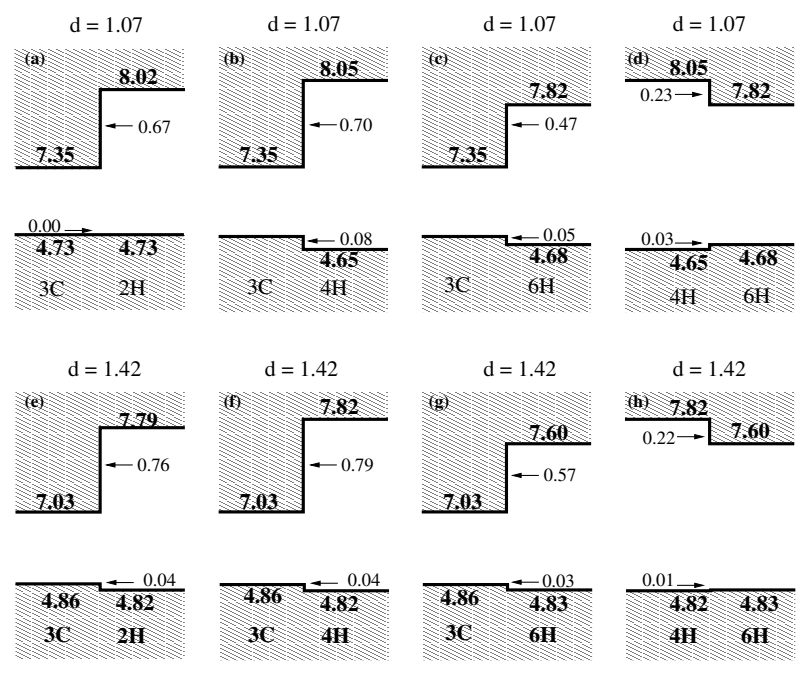

FIG. 5. Schematic model of $(\mathrm{a}+\mathrm{e}) 3 \mathrm{C} / 2 \mathrm{H}-,(\mathrm{b}+\mathrm{f}) 3 \mathrm{C} / 4 \mathrm{H}-$ , $(\mathrm{c}+\mathrm{g}) 3 \mathrm{C} / 6 \mathrm{H}-$, and $(\mathrm{d}+\mathrm{h}) 4 \mathrm{H} / 6 \mathrm{H}-\mathrm{SiCNW}$ somojunctions. Top panels: $d \approx 1.07 \mathrm{~nm}$ for $3 \mathrm{C}-, 2 \mathrm{H}-, 4 \mathrm{H}-$, and $6 \mathrm{H}-\mathrm{SiCNW}$. Bottom panels: $d \approx 1.42 \mathrm{~nm}$ for $3 \mathrm{C}-, 2 \mathrm{H}-, 4 \mathrm{H}-$, and $6 \mathrm{H}-$ SiCNWs.

$2 \mathrm{H}$ - and 3C-SiCNWs have the same energy, while the $\mathrm{CBM}$ of $2 \mathrm{H}$ lies $0.67 \mathrm{eV}$ above the CBM of $3 \mathrm{C}-\mathrm{SiCNW}$, resulting in a valence (conduction) band offset of 0.00 $(0.67) \mathrm{eV}$, as depicted in Fig. 5(a). We noticed, additionally, that there is a dependence between the band offsets and the NW diameters: by increasing the diameter of $3 \mathrm{C} / 2 \mathrm{H}-\mathrm{SiCNW}$ from 1.07 to $1.42 \mathrm{~nm}$, the valence and conduction band offsets increase from 0.00 to $0.04 \mathrm{eV}$ and from 0.67 to $0.76 \mathrm{eV}$, respectivelly [see Figs. 5(a) and (e)], giving rise to a type-I band alignment. As we mentioned in the previous paragraph, an experimental study by Kuang and $\mathrm{CaO}^{59}$ reported type-II band offset for thick (50-200 nm) 3C/2H-SiCNW homojunctions; this suggests that $\mathrm{SiC}$ NWs may have the same behavior as observed for cubic/hexagonal Si NW homojunctions, ${ }^{62}$ with type-I alignment for thin nanowires and type-II band offset for thick nanowires. Further investigations are necessary to clarify the dependence of band offsets, in SiCNW homojunctions, with (i) the NW diameter (by considering larger diamenters), and (ii) the width of the different stacking regions.

We are aware that this an estimation since we are not taking into account the charge transfers (dipole effects) at the interfaces. Such a charge transfer should be small since we have the same atomic elements at the both sides of the interface. In this case, the intrinsic properties of the bulk phase, viz.: energy band-gap, ionization potential, and electronic affinity, will play the major role in the band alignment. Thus, the general trends of the band offsets, for different polytype combinations, can be inferred through the electronic properties of the separated NWs.

Further VBM/CBM energy level comparison allows us to infer that the $3 \mathrm{C} / 4 \mathrm{H}-$ and $3 \mathrm{C} / 6 \mathrm{H}-\mathrm{SiCNW}$ will present a type-I band alignment, Figs. $5(\mathrm{~b}+\mathrm{f})$ and $5(\mathrm{c}+\mathrm{g})$, with 
both electrons and holes confined in the 3C-SiCNW. Those predictions are reasonable, since the energy gaps of $4 \mathrm{H}-$, and $6 \mathrm{H}-\mathrm{SiC}$ bulk phase are larger when compared with the one of $3 \mathrm{C}-\mathrm{SiC}$ bulk. Finally, by combining the $4 \mathrm{H}$ and $6 \mathrm{H}$ stacking, Figs. $5(\mathrm{~d})$ and $(\mathrm{h})$, we can infer an electron localization in the $6 \mathrm{H}$ region $(\mathrm{CBM} \approx 0.2 \mathrm{eV})$, and delocalized hole states $(\mathrm{VBM} \approx 0)$.

\section{SUMMARY}

In summary, we performed first-principles calculations to investigate the effects of the diameter on the mechanical and electronic properties of hydrogen-passivated [111] oriented 3C-, and [0001] oriented 2H-, 4H-, and 6HSiCNWs. Our results show that the Young's moduli and the band gaps of the nanowires are larger than those of their counterpart bulk phases, and decrease with the increasing diameter. All nanowires exhibit direct band gaps, in contrast with the indirect band gaps observed in bulk $\mathrm{SiC}$, and $3 \mathrm{C}-\mathrm{SiCNW}$ have the narrowest band gap among the nanowires investigated. We also examined the effect of uniaxial stress on the electronic properties of these nanowires; our results reveal that the band-gap dependence on the strain is different for each nanowire type. Under tensile stress we found, for example, that the band gaps of 3C-SiCNWs increase while the band gaps of $4 \mathrm{H}-\mathrm{SiCNW}$ with larger diameters decrease. Finally, comparing the energy positions of the valence band maximum and the conduction band minimum of the SiCNWs with different polytypes, we estimate the band offsets for homostructures of SiCNWs. We found that $3 \mathrm{C} / 4 \mathrm{H}$ and $3 \mathrm{C} / 6 \mathrm{H}$ SiCNWs homostructures present a type-I band alignment, with both electrons and holes lying in the $3 \mathrm{C}$ SiCNW layers.

\section{ACKNOWLEDGMENTS}

All calculations were performed using the computational facilities of CENAPAD/SP, Brazil. J.B.O. and R.H.M. acknowledge financial support from the Brazilian agencies CNPq and FAPEMIG.

\footnotetext{
${ }^{1}$ R. Wu, K. Zhou, C. Y. Yue, J. Wei, and Y. Pan, Progress in Materials Science 72, 1 (2015)

${ }^{2}$ H. bo Lu, B. C. Y. Chan, X. Wang, H. T. Chua, C. L. Raston, A. Albu-Yaron, M. Levy, R. Popowitz-Biro, R. Tenne, D. Feuermann, and J. M. Gordon, Nanotechnology 24, 335603 (2013)

${ }^{3}$ Z. Pan, H.-L. Lai, F. C. K. Au, X. Duan, W. Zhou, W. Shi, N. Wang, C.-S. Lee, N.-B. Wong, S.-T. Lee, and S. Xie, Adv. Mater. 12, 1186 (2000)

${ }^{4}$ G. Shen, D. Chen, K. Tang, Y. Qian, and S. Zhang, Chem. Phys. Lett. 375, 177 (2003)

${ }^{5}$ W. Zhou, X. Liu, and Y. Zhang, Appl. Phys. Lett. 89, 223124 (2006)

${ }^{6}$ J. J. Niu and J. N. Wang, J. Phys. Chem. B 111, 4368 (2007)

${ }^{7}$ G. Z. Yang, H. Cui, Y. Sun, L. Gong, J. Chen, D. Jiang, and C. X. Wang, J. Phys. Chem. C 113, 15969 (2009)

${ }^{8}$ X. Luo, W. Ma, Y. Zhou, D. Liu, B. Yang, and Y. Dai, Nanoscale Res. Lett 5, 252 (2010)
}

${ }^{9}$ H.-K. Seong, H.-J. Choi, S.-K. Lee, J.-I. Lee, and D.-J. Choi, Appl. Phys. Lett. 85, 1256 (2004)

${ }^{10}$ K.-Z. Li, J. Wei, H.-J. Li, Z.-J. Li, D.-S. Hou, and Y.-L. Zhang, Mat. Sci. Eng. A 460-461, 233 (2007)

${ }^{11}$ J. Wei, K. Li, H. Li, D. Hou, Y. Zhang, and C. Wang, J. Alloys Compd. 462, 271 (2008)

${ }^{12}$ D.-W. Kim, Y.-J. Choi, K. J. Choi, J.-G. Park, J.-H. Park, S. M. Pimenov, V. D. Frolov, N. P. Abanshin, B. I. Gorfinkel, N. M. Rossukanyi, and A. I. Rukovishnikov, Nanotechnology 19, 225706 (2008)

${ }^{13}$ Y. Yang, G. Meng, X. Liu, L. Zhang, Z. Hu, C. He, and Y. Hu, J. Phys. Chem. C 112, 20126 (2008)

${ }^{14}$ E. W. Wong, P. E. Sheehan, and C. M. Lieber, Science 277, 1971 (1997)

${ }^{15}$ X. D. Han, Y. F. Zhang, K. Zheng, X. N. Zhang, Z. Zhang, Y. J. Hao, X. Y. Guo, J. Yuan, , and Z. L. Wang, Nano Lett. 7, 452 (2007)

${ }^{16}$ W. Yang, H. Araki, C. Tang, S. Thaveethavorn, A. Kohyama, H. Suzuki, and T. Noda, Advanced Materials 17, 1519 (2005)

${ }^{17}$ W. Nhuapeng, W. Thamjaree, S. Kumfu, P. Singjai, and T. Tunkasiri, Current Applied Physics 8, 295 (2008), aMN3 (Third International Conference on Advanced Materials and Nanotechnology)Third International Conference on Advanced Materials and Nanotechnology

${ }^{18}$ R. Rurali, Phys. Rev. B 71, 205405 (2005)

${ }^{19}$ More than 200 polytypes are known for bulk SiC. The most studied ones are the hexagonal polytypes $2 \mathrm{H}, 4 \mathrm{H}, 6 \mathrm{H}$, and the cubic form $3 \mathrm{C}$ (or $\beta$ ).

${ }^{20}$ M. Durandurdu, Phys. Stat. Sol. (b) 243, R37 (2006)

${ }^{21}$ B. Yan, G. Zhou, W. Duan, J. Wu, and B.-L. Gu, Appl. Phys. Lett. 89, 023104 (2006)

${ }^{22}$ Z. Wang, M. Zhao, T. He, H. Zhang, X. Zhang, Z. Xi, S. Yan, X. Liu, and Y. Xia, J. Phys. Chem. C 113, 12731 (2009)

${ }^{23}$ P. Hohenberg and W. Kohn, Phys. Rev. 136, B864 (1964)

${ }^{24}$ J. M. Soler, E. Artacho, J. D. Gale, A. García, J. Junquera, P. Ordejón, and D. Sánchez-Portal, J. Phys.: Condens. Matter 14, $2745(2002)$

${ }^{25}$ N. Troullier and J. L. Martins, Phys. Rev. B 43, 1993 (1991)

${ }^{26}$ W. Kohn and L. J. Sham, Phys. Rev. 140, A1133 (1965)

${ }^{27}$ D. M. Ceperley and B. J. Alder, Phys. Rev. Lett. 45, 566 (1980)

${ }^{28}$ O. F. Sankey and D. J. Niklewski, Phys. Rev. B 40, 3979 (1989)

29 J. J. Petrovic, J. V. Milewski, D. L. Rohr, and F. D. Gac, Petrovic 20, 1167 (1985)

${ }^{30}$ W. R. Lambrecht, B. Segall, M. Methfessel and, M. Van Schilfgaarde, Phys. Rev. B 44, 3685 (1991)

${ }^{31}$ G. L. Harris, Properties of Silicon Carbide (Institution of Engineering and Technology, London, 1995)

${ }^{32}$ W. A. Harrison, Electronic Structure and the Properties of Solids (Dover, 1989)

${ }^{33}$ O. Auciello, J. Birrell, J. A. Carlisle, J. E. Gerbi, X. Xiao, B. Peng, and H. D. Espinosa, J. Phys.: Condens. Matter 16, R539 (2004)

${ }^{34}$ P. Giannozzi, S. Baroni, N. Bonini, M. Calandra, R. Car, C. Cavazzoni, D. Ceresoli, G. L. Chiarotti, M. Cococcioni, I. Dabo, A. D. Corso, S. de Gironcoli, S. Fabris, G. Fratesi, R. Gebauer, U. Gerstmann, C. Gougoussis, A. Kokalj, M. Lazzeri, L. Martin-Samos, N. Marzari, F. Mauri, R. Mazzarello, S. Paolini, A. Pasquarello, L. Paulatto, C. Sbraccia, S. Scandolo, G. Sclauzero, A. P. Seitsonen, A. Smogunov, P. Umari, and R. M. Wentzcovitch, Journal of Physics: Condensed Matter 21, 395502 (2009)

${ }^{35}$ J. J. Petrovic, J. V. Milewski, D. L. Rohr, and F. D. Gac, J. Mater. Sci. 20, 1167 (1985)

${ }^{36}$ S. Limpijumnong and W. R. L. Lambrecht, Phys. Rev. B 57, 12017 (1998)

${ }^{37}$ C. H. Park, B.-H. Cheong, K.-H. Lee, and K. J. Chang, Phys. Rev. B 49, 4485 (1994)

${ }^{38}$ K. J. Chang and M. L. Cohen, Phys. Rev. B 35, 8196 (1987)

${ }^{39}$ M. Jiang, S. M. Peng, H. B. Zhang, C. H. Xu, H. Y. Xiao, F. A. Zhao, Z. J. Liu, and X. T. Zu, Sci. Rep. 6 (2016), 
$10.1038 /$ srep20669

${ }^{40}$ I. S. Santos de Oliveira and R. H. Miwa, Phys. Rev. B 79, 085427 (2009)

${ }^{41}$ C. Persson and U. Lindefelt, Phys. Rev. B 54, 10257 (1996)

${ }^{42}$ Z. Wang, M. Zhao, T. He, X. Zhang, Z. Xi, S. Yan, X. Liu, and Y. Xia, J. Phys. Chem. C 113, 856 (2009)

${ }^{43}$ T. Ito, K. Sano, T. Akiyama, and K. Nakamura, Thin Solid Films 508, 243 (2006)

${ }^{44} \mathrm{G}$. Wang and X. Li, Journal of Applied Physics 104, 113517 (2008)

${ }^{45}$ S. Cuenot, C. Frétigny, S. Demoustier-Champagne, and B. Nysten, Phys. Rev. B 69, 165410 (2004)

${ }^{46}$ Y. Chen, T. Burgess, X. An, Y.-W. Mai, H. H. Tan, J. Zou, S. P. Ringer, C. Jagadish, and X. Liao, Nano Letters 16, 1911 (2016), pMID: 26885570, http://dx.doi.org/10.1021/acs.nanolett.5b05095

${ }^{47}$ C. L. dos Santos and P. Piquini, Phys. Rev. B 81, 075408 (2010)

${ }^{48}$ P. W. Leu, A. Svizhenko, and K. Cho, Phys. Rev. B 77, 235305 (2008)

${ }^{49}$ B. Lee and R. E. Rudd, Phys. Rev. B 84, 161303 (2011)

${ }^{50}$ A. J. Lee, M. Kim, C. Lena, and J. R. Chelikowsky, Phys. Rev. B 86, $115331(2012)$

${ }^{51}$ M. A. Makeev, D. Srivastava, and M. Menon, Phys. Rev. B 74, $165303(2006)$
${ }^{52}$ P. W. Leu, A.Svizhenko, and K.Cho, Phys. Rev. B 77, 235305 (2008)

${ }^{53}$ A. Fissel, Physics Reports 379, 149 (2003)

${ }^{54}$ A. Qteish, V. Heine, and R. J. Needs, Phys. Rev. B 45, 6534 (1992)

${ }^{55}$ K. F. Dombrowski et. al., Appl. Phys. Lett. 65, 1811 (1994)

${ }^{56}$ A. O. Evwaraye, S. R. Smith, and W. C. Mitchel, Appl. Phys. Lett. 67, 3319 (1995)

${ }^{57}$ S. P. Beckman, J. Han, and J. R. Chelikowsky, Phys. Rev. B 74, 165314 (2006)

${ }^{58}$ H. Zhang, Y. Xu, J. Zhou, J. Jiao, Y. Chen, H. Wang, C. Liu, Z. Jiang, and Z. Wang, J. Mater. Chem. C 3, 4416 (2015)

${ }^{59}$ J. Kuang, and W. Cao, Appl. Phys. Lett. 103, 112906 (2013)

${ }^{60}$ K. Pemasiri, M. Montazeri, R. Gass, L. M. Smith, H. E. Jackson, J. Yarrison-Rice, S. Paiman, Q. Gao, H. H. Tan, C. Jagadish, X. Zhang, and J. Zou, Nano Letters 9, 648 (2009), pMID: 19170615, http://dx.doi.org/10.1021/nl802997p

${ }^{61}$ M. D. Moreira, P. Venezuela, and R. H. Miwa, Nanotechnology 21, $285204(2010)$

${ }^{62}$ M. Amato, T. Kaewmaraya, A. Zobelli, M. Palummo, and R. Rurali, Nano Letters 16, 5694 (2016) 